\title{
ESTUDIOS SOBRE LAS RUBIÁCEAS DE MÉXICO, LII Una especie nueva del género Randia (Gardenieae, Rubiacae) en Chiapas
}

\author{
A. Borhidi ${ }^{1}$ y E. Martínez SAlas ${ }^{2}$ \\ ${ }^{1}$ Departamento de Fitotaxonomía y Geobotánica, Instituto de Biología, Universidad de Pécs \\ H-7624 Pécs, Ifjúság útja 6, Hungría; E-mail: borhidi@gamma.ttk.pte.hu \\ ${ }^{2}$ Instituto de Biología, Universidad Autónoma de México (UNAM), México \\ E-mail:ems@ib.unam.mx
}

(Received 4 November, 2014; Accepted 15 December, 2014)

The Randia sepium is an extremely interesting species with its coriaceous medium-sized leaves glabrous and brilliant on both sides with 4-7 pairs of secondary nerves and terminal inflorescences, with medium-sized hirsute flowers and pubescent corolla lobes on both sides not having closely related taxon in the Mexican and Mesoamerican flora. All Randia species of these areas having coriaceous shiny leaves - e.g. R. cookii and R. chiapensis - are thorny small-leaved, nerveless, small-flowered ones with small glabrous, axillary or terminal solitary flowers. It is notable for its use, since it is applied as living fence in Chiapas (Mexico).

Key words: fence, Chiapas, México, Randia, Rubiaceae

\section{Randia sepium Borhidi et E. Martínez, spec. nova, hoc loco}

(Fig. 1)

Tipo: México, Chiapas, Mpio. Ocosingo, Comunidad Lacandona de Lacanhá-Chansayab, a $130 \mathrm{~km}$ al sureste de Palenque por la carretera fronteriza hasta el crucero San Javier, después $8 \mathrm{~km}$ hacia el Oeste. Bosque tropical perennifolio. Suelos rendzinas y litosoles. Coord.: $16^{\circ} 44^{\prime}$ N, $91^{\circ} 05^{\prime}$ W, alt. 400 m snm. Col.: Samuel Lewy T. y Alejandro Durán F. (439), 25.05.1995. Holotipo: MEXU. Uso: se usa como cerco vivo sembrándolo por estaca.

Arbor parva inermis $5 \mathrm{~m}$ alta, rami cylindracei, glabri, longitudinaliter striati superficie angulato-cristata, transversaliter fissurata, internodiis brevibus, 3-5 $\mathrm{mm}$ longis. Stipulae terminales late triangulares, ovatae vel lanceolatae, 2.5-3 mm longae et $4 \mathrm{~mm}$ latae, in mucronem 2-3 mm longam terminatae, extus adpresse strigosae vel glabrescentes usque glabrae, solummodo basi strigosae, intus basi dense strigosae et ad marginem strigulosae. Stipulae interpetiolares glabrae, rane caducae, mucronulatae, 
adaxialiter dense strigillosae. Folia lanceolata, oblanceolata vel anguste elliptica, 4-8 $\mathrm{cm}$ longa et 1-1.7 cm lata, basi apiceque longe attenuata, essentialiter glabra, apice acuta, basi longe cuneata, sessilia vel subsessilia, petiolis 0-3 mm longis; limbus folii coriacei vel subcoriacei, viridis, nitidulus, concoloris, nervo medio supra prominulo, subtus prominente, lateralibus utroque latere 4-7, supra obsoletis vel inconspicuis, subtus prominentibus, apicem versus abrupte arcuatis, ante marginem plerumque non connectis, nervatura roseo-flavescens, margine integro leviter recurvo, sparse glanduloso-punctato. Inflorescentiae terminales, capitatae, sessiles, 1-3-florae. Flores bisexuales, albi. Hypanthium oblongo-obovatum, 6-7 mm longum et apice 4-4.5 $\mathrm{mm}$ latum, basi acutum, patenti-hirsutum; limbus calycis nullus, lobi 5, linear-lanceolati, dorso costati, aequilongi, usque ad $10 \mathrm{~mm}$ longi et basi 1.5-2 $\mathrm{mm}$ lati, utrinque hirsuti. Corolla alba, tubus corollae 15-20 mm longus, extus sericeo-hirsutus, intus glaber; lobi 5, late ovati vel elliptici, 12-15 mm longi et 7-8 $\mathrm{mm}$ lati, apice breviter apiculati, utrinque dense pilosi, et intus basi hirsuti, sed faux glabra. Stamina 5, sessiles $0.5-1 \mathrm{~mm}$ sub orificio tubi, antherae lineares vel oblongo ellipticae, $4.5-5 \mathrm{~mm}$ longae et $1 \mathrm{~mm}$ latae, apice curvatae medio superiori e fauce exserto. Pistillum breve et crassum, 10-12 mm longum, medio tubi insertum. Stylus $4 \mathrm{~mm}$ longus, crassus, angulatus sparse pilosus. Stigma linear-ellipticum, 5-5.5 mm longum, sparse pilosulum. Ovarium oblongo obovatum, apice e lobulis calycis breviter, 1-1.5 mm longe elevato et hirsuto, 2-locular; ovula numerosa ad septum in lineam centralem affixa. Fructus ignotus.

Árbol inerme de $5 \mathrm{~m}$ de altura, ramas cilíndricas, glabras, longitudinalmente estriadas formando una superficie anguloso-crestada, transversalmente fisuradas, entrenudos cortos de 3 a $5 \mathrm{~mm}$ de largo. Estípulas terminales anchamente triangulares, ovadas o lanceoladas, de 2.5 a $3 \mathrm{~mm}$ de largo y 4 $\mathrm{mm}$ de ancho, apretado estrigosas o glabrescentes a glabras por fuera, excepto la base estrigosa, densamente apretado estrigosas por dentro con un mucrón terminal de 2 a $3 \mathrm{~mm}$ de largo, estrigoso en el margen. La estípula interpeciolar triangular, glabra, caediza, mucronulada, densamente apretado estrigillosa adaxialmente. Hojas lanceoladas u oblanceoladas o estrechamente elípticas de 4 a $8 \mathrm{~cm}$ de largo y 1 a $1.7 \mathrm{~cm}$ de ancho, largamente atenuadas en ambos extremos, esencialmente glabras, agudas en el ápice, largamente cuneadas en la base, sésiles o subsésiles con pecíolos alados de 0-3 $\mathrm{mm}$ de largo; el limbo de la hoja verde, nitídulo, concolor, nervio medio promínulo en el haz, prominente en el envés, los laterales 4-7 pares obsoletos o inconspicuos en el haz, prominentes en el envés fuertemente arqueados hacia el ápice y mayormente no se conectan antes del margen, la nervadura de color rojízo-amarillenta, glabra, coriáceas a subcoriáceas, margen entero, poco recurvo, esparcidamente glandular-punteado. Inflorescencias terminales, 1-3-flores, capitadas, sésiles. Flores hermafroditas, blancas. Hipanto oblongo-obovado, de 6 a $7 \mathrm{~mm}$ de lar- 


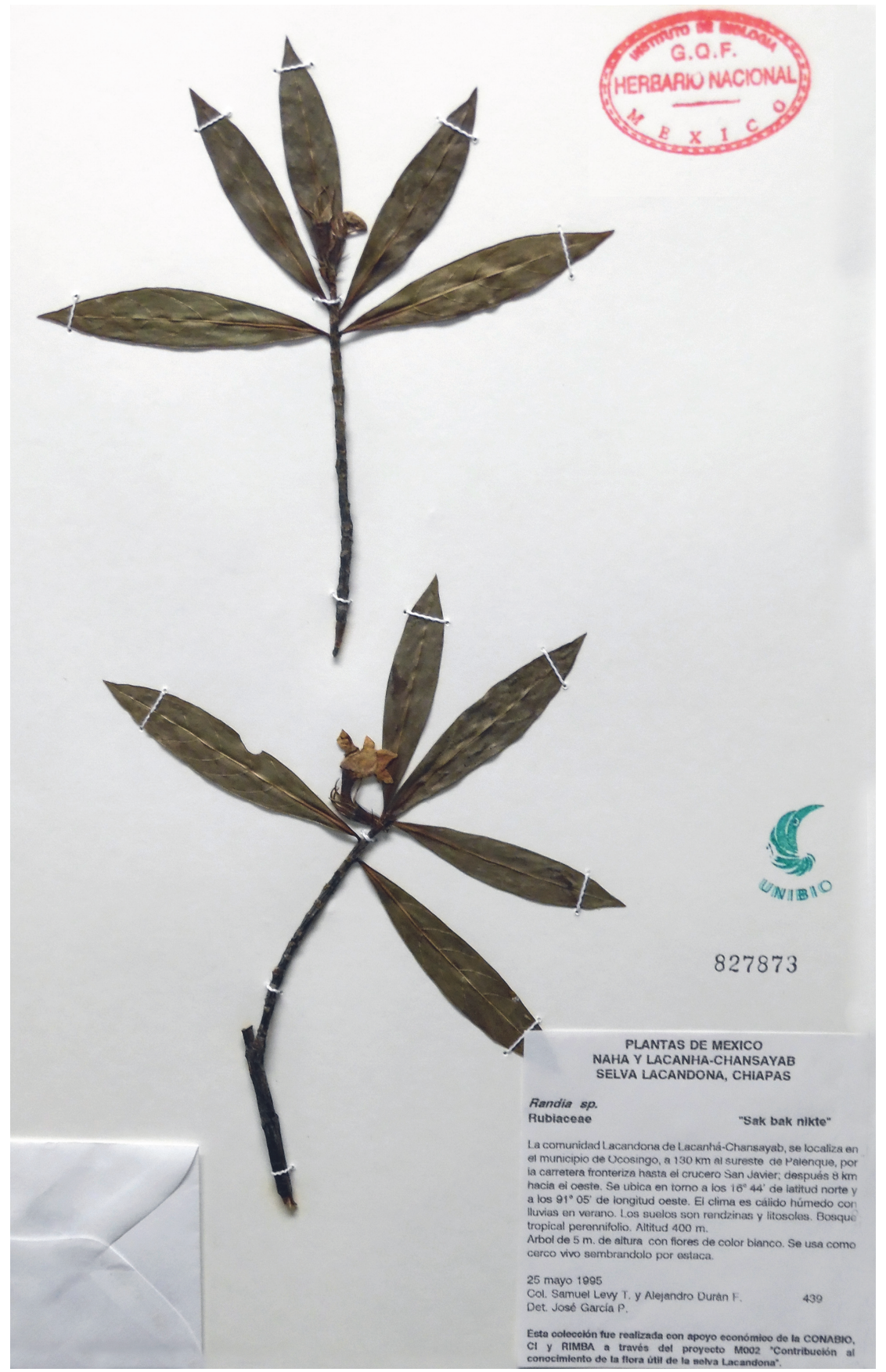

Fig. 1. Holotipo de Randia sepium Borhidi et E. Martínez en MEXU (Foto: A. Borhidi) 
go y 4 a $4.5 \mathrm{~mm}$ de ancho en el ápice, agudo en la base extendido hirsuto, limbo del cáliz nulo, lóbulos 5, linear-lanceolados con una costa dorsal, iguales, de $10 \mathrm{~mm}$ de largo y 1.5 a $2 \mathrm{~mm}$ de ancho en la base, hirsutos en ambas caras. Corola blanca, tubo corolino de 15 a $20 \mathrm{~mm}$ de largo, seríceo-hirsuto por fuera, glabro por dentro; lóbulos 5, anchamente ovados a elípticos de 12 a $15 \mathrm{~mm}$ de largo y 7 a $8 \mathrm{~mm}$ de ancho, brevemente apiculados en el ápice, densamente pilosos en ambas caras y hirsutos en la base por dentro, pero la garganta es glabra. Estambres 5, sésiles en 0.5 a $1 \mathrm{~mm}$ debajo del orificio del tubo, anteras lineares u oblongo elípticas, de 4.5 a $5 \mathrm{~mm}$ de largo y $1 \mathrm{~mm}$ de ancho, encorvadas en el ápice, la mitad superior exerta da la garganta. Pistilo corto y grueso, de 10 a $12 \mathrm{~mm}$ de largo, incluso en la mitad del tubo. Estilo de $4 \mathrm{~mm}$ de largo, grueso, de $0.5 \mathrm{~mm}$ de ancho, anguloso, glabro; estigma linear-elíptico, de 5 a $5.5 \mathrm{~mm}$ de largo ambos esparcido pelosos. Ovario oblongo-obovado, el ápice elevado del cáliz en 1-1.5 mm de largo, hirsuto, 2-locular; óvulos numerosos a lo largo de una linea central de la pared del septo. Fruto desconocido.

Etimología: El epiteto específico tiene origen latino: sepes, sepium que es: cerco, refiriendose a la observación de los colectores, que la planta esta utilizada como cerco vivo sembrándolo por estaca. Otros ejemplos: Glyricidia sepium, Vicia sepium (Fabaceae).

Es una especie interesantísima, aparentemente aislada en sentido taxonómico en las areas de la flora Mexicana y Mesoamericana, con sus hojas coriáceas, glabras y lustrosas de medio tamaño, con nervios secundarios 4-7 pares e inflorescencia terminal con flores de medio tamaño densamente hirsutas en el tubo por fuera y lóbulos de la corola pubescentes en ambas caras, mientras las especies más estrechamente relacionadas de las areas mencionadas por sus hojas coriáceas y lustrosas - como p. e. R. cookii y R. chiapensis - son plantas espinosas micrófilas, con flores pequeñas, solitarias, glabras y hojas con con nervadura inconspícua. La especie es sobresaliente también por su uso insólito en el género Randia, que según la observación de los colectores, la especie esta utilizado en Chiapas como cerco vivo sembrándolo por estaca.

Agradecimientos - La colección fue realizada con apoyo económico de la CONABIO, CI y RIMBA a través del proyecto M002 “Contribución al conocimiento de la flora útil de la selva Lacandona". Los autores expresan sus agradecimientos al Dr. Gerardo Adolfo Salazar, jefe del Departamento de Botánica, del Instituto de Biologia de la UNAM y al Dr. David Sebastian Gernandt, jefe del Herbario Nacional de México (MEXU) por su apoyo en crear condiciones favorables para este trabajo. 


\section{LITERATURA CONSULTADA}

Borhidi, A. (2012): Rubiáceas de México. Segunda y ampliada edición. - Akadémiai Kiadó, Budapest, $610 \mathrm{pp}$.

Borhidi, A. y Martínez Salas, E. (2011): Estudios sobre Rubiáceas Mexicanas XXX. Tres especies nuevas y un nombre nuevo en el género Randia. - Acta Bot. Hung. 53: 31-40. doi: 10.1556/ABot.53.2011.1-2.3

Borhidi, A. y Salas-Morales, S. (2009): Estudios sobre Rubiáceas Mexicanas XXIII. Dos especies nuevas del género Randia L. en Oaxaca. - Acta Bot. Hung. 51: 21-25. doi: 10.1556/ ABot.51.2009.1-2.4

Borhidi, A., Diego-Pérez, N. y Saynes, A. (2006): Estudios sobre Rubiáceas Mexicanas, IX. Tres especies nuevas en el género Randia L. (Gardenieae) en Guerrero y Oaxaca. Acta Bot. Hung. 48: 47-50. doi: 10.1556/ABot.48.2006.1-2.6

Borhidi, A., García-Gonzalez, I. y Martínez Salas, E. (2007): Estudios sobre Rubiáceas Mexicanas XIV. Tres taxa nuevos del género Randia L. - Acta Bot. Hung. 49: 53-57. doi: 10.1556/ABot.49.2007.1-2.5

Borhidi, A., Martínez Salas, E. y Salas-Morales, S. (2012): Estudios sobre Rubiáceas Mexicanas XXXVII. Dos especies nuevas del género Randia L. (Gardenieae) en Oaxaca y Veracruz. - Acta Bot. Hung. 54: 59-66. doi: 10.1556/ABot.54.2012.1-2.6

Borhidi, A., Martínez Salas, E. y Salas-Morales, S. (2013): Estudios sobre Rubiáceas Mexicanas XLI. Tres nuevas especies del género Randia L. - Acta Bot. Hung. 55: 17-25. doi: 10.1556/ABot.55.2013.1-2.2

Lorence, D. H. (2012): Randia L. - In: Davidse, G., Sousa, M., Knapp, S. y Chiang, F. (eds gen.): Flora Mesoamérica. IV(2): Rubiaceae, Cordiaceae, Boraginaceae, Lamiaceae. Universidad Nacional Autónoma de México, Missouri Botanical Garden, The Natural History Museum (London), pp. 241-253.

Stranczinger, Sz., Borhidi, A., Szentpéteri, J. L. and Jakab, F. (2007): The phylogenetic relationships among some Randia (Rubiaceae) taxa. - Acta Biol. Hung. 58: 235-244. doi: 10.1556/ABiol.58.2007.2.10 